\title{
Compensation of the Aperture Ratio Markings of a Photographic Lens for Absorption, Reflection, and Vignetting Losses
}

\author{
By Irvine C. Gardner
}

\begin{abstract}
At present the diaphragm markings of a photographic lens are based entirely upon geometrical considerations and do not take into account the losses of light resulting from absorption, reflection, and scattering. A method of equivalent marking is described in which, for example, the marking 8 does not correspond to the geometrically determined aperture ratio $1: 8$ but to an opening sufficiently larger to permit the transmission of as much light as would be transmitted by the aperture 1:8 in the absence of any losses due to absorption, etc. Such a system of apertures may be referred to as equivalent, or compensated, apertures. Two systems of compensation are given, one based upon the illumination at the center of the field and the other based upon the average illumination over the entire field, thus taking vignetting into account.

A relatively simple photometric procedure for determining either of the two systems of compensated graduations is described. For use during a transition period, a system of markings is described that will permit exposures to be determined either with light losses compensated or by the present method without compensation. Except for the change of markings on a lens, no other instrumental changes are required to apply the new system of exposure determination.
\end{abstract}

\section{Introduction}

Photography is both an art and a technic. Until a few years ago it was largely empirical, each photographer's practice being based on his own experience with his own particular equipment. Now, however, the principles underlying photographic work are so well known, and quantitative relations are so precisely established, that photographic engineering may be said to have become an applied science. The possibility of saving large sums of money and at the same time securing greater uniformity of result by greater precision in the exposing and processing of the very large quantity of film used for motion picture photography has constituted an economic urge in favor of this transformation. The development of the modern photoelectric exposure meter and the more general dissemination of quantitative information regarding the properties of emulsions have made it possible not only for the professional, but also for the skilled amateur to control his photographic work in a scientific manner.

With this progress a demand has arisen for a more scientific method of marking the diaphragm openings of a lens. The method now in general use is based entirely upon the diameter of the entrance pupil and the equivalent focal length. 
It gives no consideration to the loss of effective light that arises because of absorption in the glasses of which the lens elements are made or because of reflection and scattering at the surfaces. Photographic objectives in common use may have from 4 to 10 glass-air surfaces. The transmissions of such lenses may range from 50 to 80 percent if uncoated and may be as high as 95 percent if efficient low-reflection coatings are applied to the surfaces. It is evident, therefore, that the effective exposures for different lenses with the iris diaphragm set for the same aperture ratio, may vary almost by a factor of 2 , an uncertainty that is inconsistent with the precision with which the other factors governing exposure are controlled. Several methods for calibrating and marking the apertures of a photographic lens that will effect a correction for the varying light losses with different lenses have been proposed.

This publication presents a method that is relatively simple and direct, and by which different laboratories may be expected to arrive at the same system of marking and equivalent values without the interchange of physical standards. The method is extended to apply to lenses when focused for infinity or for any finite distance, and a system for marking the diaphragm scale is suggested. In order to distinguish between the older and newer systems of marking, the aperture ratio of the older system, based on geometrical measurements only, will be referred to as the geometrical aperture ratio. The new aperture ratio, which takes the absorption and reflection losses into consideration will, for present purposes, be designated the equivalent aperture ratio. Other terms that might be used are compensated aperture ratio, and $t$-aperture ratio, the $t$ standing for transmission as suggested by Berlant. ${ }^{1}$ Consideration is also given to the difference in performance of different lenses because of vignetting.

\section{Method of Calibration for Infinite Object Distance}

In figure $1, L$ represents a photographic lens that receives light from the uniformly illuminated screen $C D$. In the focal plane there is the plate $G H$, with the aperture of area $A$ centered at

1 E. Berlant, Lens stop calibration by transmission, J. Soc. Motion Picture Engrs. 46, 17 (1946); C. R. Daily, A lens calibrating system, J. Soc. Motion Picture Engrs. 46, 343 (1946); A. E. Murray, The photometric calibration of lens apertures, J. Soc. Motion Picture Engrs. 46, 142 (1946).
0 . Behind this aperture there is a photoelectric cell with receiver $R$, which is large enough to receive all the luminous flux from screen $C D$ that is transmitted by the photographic lens and the aperture at $O$. The full lines represent the boundary of the cone of rays proceeding from the screen to the axial point of the focal plane. In the object space the cone degenerates into a cylinder, of which the diameter is $d$. In the image space the half-angle of the cone is $\alpha$, where $\alpha$ is defined by the equation ${ }^{2}$

$$
\sin \alpha=d / 2 f \text {. }
$$

\section{\begin{tabular}{l}
$C$ \\
\hline \\
\hline
\end{tabular}}

FIGURE 1.-Arrangement of apparatus for measurement of total luminous flux transmitted through a photographic objective and through an aperture of area $A$ in the focal plane at 0 .

The geometrical aperture ratio is $1: 1 /(2 \sin \alpha)$.

The aperture ratio of the lens, for an infinitely distant object, is $1: f / d$, and $f / d$ is the $f$-number. It is evident that

$$
f \text {-number }=\frac{1}{2 \sin \alpha}
$$

In figure 2, $C D$ represents a uniformly illuminated screen identical with that of figure 1 . Screen $G H$ with aperture, of area $A$ at $O$, and photoelectric cell with receiver $R$ are identical with the similar parts of figure 1 . At $I J$, at a distance $e$ from the plane $G H$, there is a circular diaphragm of diameter such that the angle, $\alpha$, in figure 2 equals the corresponding angle of figure 1. For the moment it will be assumed that the length, $e$, is identical with the equivalent focal length of the photographic objective of figure 1 . If $B$ is

2 There is a temptation to write this equation $\tan \alpha=d / 2 f$, but if the lens is suitably corrected for coma, a necessary condition for a photographic objective, eq 1 is correct. 
lent aperture ratio corresponding to the maximum indicated geometrical aperture ratio, it is necessary to secure the balance between measurements of figures 1 and 2 by adjusting the diameter of aperture $I J$ in figure 2 .

Some of the details and necessary precautions can now be profitably considered. It is not necessary that length $e$ of figure 2 be exactly equal to the focal length of the lens being tested, provided that the correct ratio is maintained between $e$ and the diameter of the aperture. It is desirable that $e$ be approximately equal (within $5 \%$ is satisfactory) in order that the approximation introduced by the finite area $A$ shall not differ greatly for the two measurements. Area $A$ must be definitely limited and, when the lens is used, it should be located accurately in the focal plane. A suitable way to achieve this is by the use of a metal plate in the focal plane with a circular aperture of the desired area and a photocell back of the aperture with a receiver large enough to receive all the light that passes through the opening. Such a plate is indicated at $G H$ in figures 1 and 2 . The dotted lines in figure 1 and 2 indicate the portion of the screen $C D$ that contributes to the illumination at $O$. It is evident that the contributing areas for the lens and for the aperture will be smaller and more nearly identical if the screen $C D$ be brought close to the lens or diaphragm respectively.

It is therefore recommended that screen $C D$ be placed immediately in front of the lens and illuminated by transmitted light. Uniform brightness of the smaller area thus utilized is more easily obtained, and deficiencies in uniformity of illumination become less important as the areas utilized during the two measurements become more nearly identical. It is, of course, essential that the brightness of the screen have the same value for the measurement with the diaphragm and with the lens. It is also assumed that all parts of screen $C D$ contributing to either measurement obey Lambert's law. In other words, a collimated beam or a surface giving specular reflection should not be used.

There are variations of the experiment that suggest themselves. For the evenly illuminated screen $C D$, an integrating sphere may be substituted. Furthermore, the directions of the light may be reversed with the source at $O$ and the receiver at $C D$. In this latter case, if $C D$ is not replaced by an integrating sphere, the receiver of the light-sensitive element must be large enough to receive all the luminous flux, and it must be uniformly sensitive over the entire area. This requirement can be most easily met if the receiver is placed near lens $L$ or aperture $I J$, as the required size is then greatly reduced.

Strictly speaking, the spectral quality of the light proceeding from source $C D$ when the measurements are made should be identical with that reflected from the object to be photographed, and the spectral sensitivity of the photocell should be identical with that of the emulsion to be used. When the great variations usually present in the spectral quality of the light proceeding to a lens from an object to be photographed are considered, it is evident that it is not practicable to fulfill this condition. Fortunately, the absorption of a photographic lens is not particularly selective for different parts of the spectrum, and the values of $k$ for a given lens will not differ greatly as the spectral distribution of the light illuminating screen $C D$ is changed. However, for precise work, standardization is desirable, and it is accordingly suggested that tungsten lamps operating at a color temperature of $2,360^{\circ} \mathrm{K}$ be used in conjunction with Wratten No. 79 filters. This gives light having a color temperature approximately that of the noon sun $\left(5,400^{\circ} \mathrm{K}\right)$. The use of a controlled source facilitates intercomparison between measurements at different laboratories. Even in the absence of such a standardized source, if screen $C D$ is illuminated by tungsten lamps operating at normal voltage, and if the photocell has a spectral sensitivity similar to that of the commercial exposure meter, the use of the equivalent aperture ratios, measured by the method of this presentation, will be much more precise and accurate than the use of the geometrical aperture ratios.

\section{Method of Indicating Equivalent Aper- ture Ratios}

According to the current method of determining aperture ratio, the diaphragm markings on the lenses will not yield consistent exposures from lens to lens. Either the speed ratings of the emulsions or the computing tables on the exposure meters, or both, may be considered as adjusted to give the correct exposure for some average value of 
$k_{0}$, typical of photographic objectives. For a lens having a value of $k_{0}$ smaller than this average value, the result will be underexposure, and for a lens having a higher value (for example, a coated lens), the result will be overexposure. If this assumed value of $k_{0}$ were known, it would be possible to alter the speed rating of the emulsions to give correct exposure with lenses graduated to read equivalent aperture ratios. To illustrate, if it were known that the value of $k_{0}$ is 0.76 , for the average lens ${ }^{4}$ is the basis on which the computation tables of a certain make of exposure meter rests, the speed rating of a photographic emulsion for use with the equivalent aperture ratios should be increased by the factor $1 / 0.76$. Once this adjustment of speed ratings has been made, exposures should be selfconsistent for all lenses graduated in terms of the equivalent aperture ratio.

Figure $3 \mathrm{~A}$ shows the diaphragm markings of a lens developed into a linear scale. Between each pair of graduations there is a dot. The indicated graduations with numbers correspond to the geometrical aperture ratios as now marked on photographic lenses. The dots correspond to the equivalent aperture ratios, each dot representing the equivalent aperture ratio of the same value as the next smaller geometric aperture ratio. To illustrate, the dot between 2.8 and 4 corresponds to the equivalent aperture ratio $1: 4$, and similarly, the dot between 5.6 and 8 corresponds to the equivalent ratio $1: 8$. Such dots in red have been used at times on photographic lenses. ${ }^{5}$

This method of marking may not be entirely unambiguous, as there is a possibility of allocating the dot to the incorrect one of the two adjacent stop numbers. Consequently, the marking shown in figure $3 \mathrm{~B}$ is suggested. In this case a line, preferably red, is drawn connecting the setting for the equivalent aperture ratio to the corresponding geometrical aperture ratio graduation. The length of this line indicates the extent to which a lens aperture must be opened beyond a given geometric aperture ratio to compensate for the loss of light resulting from reflection and absorption. Having a lens doubly marked in this manner is certainly an advantage during a period of transition when one is changing from the regular use of one set of markings to the other. In addition to marking

${ }^{4}$ W. N. Goodwin, Jr., J. Soc. Motion Picture Engrs. 20, 95 (1933).

${ }^{5}$ Paul C. Foote, of Bell \& Howell Co. has mentioned this type of marking and has made photographs available of a graduated Leica lens. the lenses, the only change required is the publication of a new set of speed ratings. During the transition period the manufacturers might well give two sets of speed ratings in their tables, one in

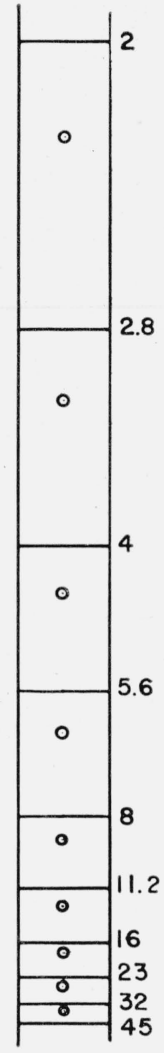

A

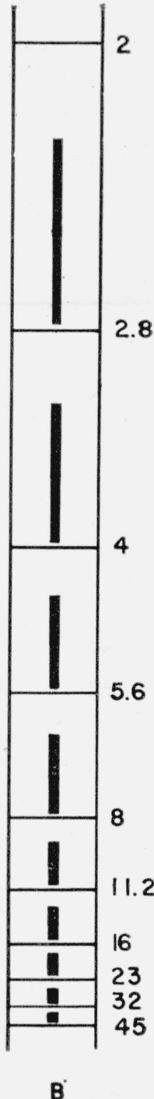

FIGURE 3.-Methods of indicating the effects of aperture ratios.

A, Aperture scale of a typical lens with the geometric aperture ratios indicated by the graduations and numbers in the conventional manner. Dots (which preferably are in red) indicate the settings for the equivalent aperture ratios. To illustrate, the dot between the graduations 5.6 and 8 corresponds to the equivalent aperture $1: 8 ; \mathrm{B}$, preferred system of marking the equivalent aperture ratios in which a line (preferably red) connects the setting for a given equivalent aperture ratio with the geometrical aperture ratio having the same numerical value.

black for use with the geometrical aperture ratios, and one in red for use with the equivalent aperture ratios. Even after the use of the equivalent aperture ratios has become general, it might well be desirable to retain the double system of marking on photographic lenses because the geometrical aperture ratios apply more precisely to the depth of focus scales with which many cameras are now provided. 


\section{Effect of Vignetting}

The measurements of section II have been concerned only with the illumination on the axis of the lens. In figure 2 , if the aperture $O$ and receiver $R$ are displaced the distance $e \tan \beta$ in a direction normal to the axis of the diaphragm, as shown in figure 4 , a measure of the flux corre-

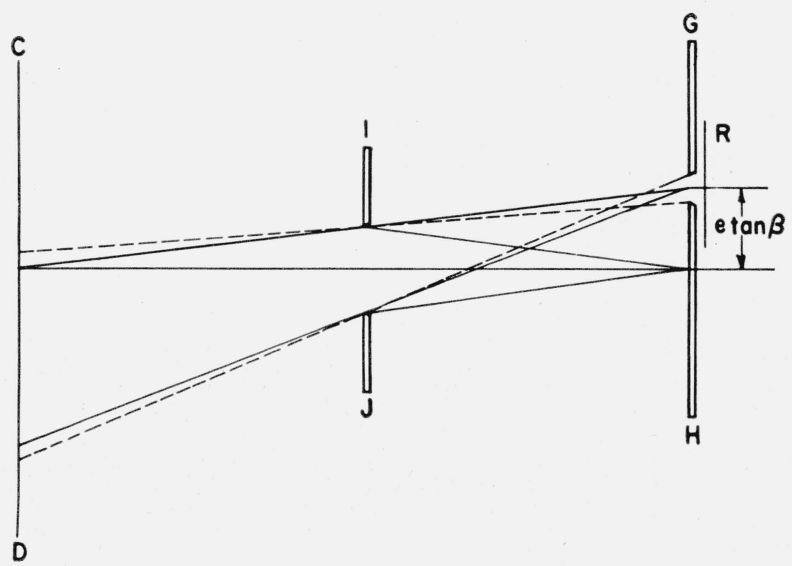

FiguRE 4.-Arrangement of apparatus for measuring the total flux transmitted through aperture $I J$ and through the aperture of area $A$ displaced from the axial position.

The measurements made with arrangements indicated in figures 2 and 4 give a measurement of the decrease of illumination for points off the axis when there is no vignetting.

sponding to an image point at an angular displacement, $\beta$, from the axis is obtained. This will be less than the axial value because of the operation of the "cosine fourth-power law," which is a statement that the illumination in the field of a photographic lens varies as the fourth power of the cosine of the angular distance from the center of the field provided that the diameters of all elements of the lens system are so great that the iris of the lens is the only part of the system that restricts the cone of transmitted rays. Although this last restriction is seldom fulfilled over the entire field of a lens, it should be noted that even when this condition is complied with, the cosine fourth-power law is an approximate rather than an exact statement.

The ratio of the illumination at the off-axis position to the axial value gives a measure of the decrease of illumination as the image point moves from the center of the field outward. Let $F_{\beta}$ and $F_{0}$ be the fluxes measured by the apparatus, as shown in figures 2 and 5 respectively. Then the ratio $F_{\beta} / F_{0}$ gives the ratio of the exposures $\beta$ degrees from the axis and on the axis for an ideal lens with no absorption and no vignetting. ${ }^{6}$

The ratio $F_{\beta} / F_{0}$ will approximately equal $\cos ^{4} \beta$. Similarly, for the arrangement of figure 1 aperture $O$ and receiver $R$ may be displaced through the distance $f \tan \beta$ and a measurement of the illumination made. It will be assumed that the iris is set for an equivalent aperture ratio the same as that for the measurements of figures 2 and 4 . We now have four readings, $F_{0}{ }^{\prime}$, the axial value for the arrangement of figure $1 ; F_{\beta}{ }^{\prime}$, the reading for the arrangment of figure 1 with the measurement made $\beta$ degrees from the axis; and $F_{0}$ and $F_{\beta}$. Since the equivalent aperture ratios are assumed to be the same in all cases, $F_{0}{ }^{\prime}=F_{0}$. The ratio $F_{\beta}{ }^{\prime} / F_{\beta}$ gives a measure of the vignetting, i. e., the falling off in illumination beyond that attributable to an ideal lens. ${ }^{7}$ However, the ratio $F_{\boldsymbol{\beta}}{ }^{\prime} / F_{0}{ }^{\prime}$ is the more useful ratio to the lens user because it gives directly the ratio of the exposure obtained at a point $\beta$ degrees from the axis to that obtained on the axis.

Different types of lenses differ greatly in the amount of vignetting. A lens system that is long in comparison with its focal length requires much larger elements than a short lens system if vignetting is to be avoided or reduced to a satisfactory value. Sometimes a lens system is such that the aberrations for the marginal parts of the field are excessive. Such a fault is rendered less apparent when there is considerable vignetting because the lens is, in effect, stopped down at the edge of the field much more than at the center. Excessive vignetting is sometimes found in folding hand cameras because the manufacturer, in order to make the camera compact, makes the lens elements small. If, for example, the lens is rated as an $f / 2$, this relative aperture may apply only at the center of the field, the exposure falling off very sharply for the corners of the picture.

\footnotetext{
6 "Vignetting" is ambiguous unless defined. It may reasonably be applied to include all the decrease of illumination that arises at an off-axis point in the image-plane of a photographic objective, or the term may be used to apply only to the decrease of illumination which arises because of restrictive action of parts of the lens mount or lens elements and which is in excess of that necessarily occurring with an ideal lens. Following the custom of several writers, the second application is used in this discussion. In accordance with this interpretation, there is no vignetting for the system of figure 4. 7 This is not strictly true, because the illumination resulting from the aperture of fig. 2 does not fall off exactly as the fourth power of the cosine. The diaphragm, however, represents a convenient standard that can be reproduced without difficulty at different laboratories and it follows the cosine fourth-power law as closely as do most lenses in common use. The approximation is better for the smaller apertures and for points near the axis. To illustrate, for points distant 40 degree from the axis, the departures are $7.6,1.8$, and 0.4 percent for the aperture ratios $1: 2,1: 4$, and $1: 8$ respectively
} 
For such a lens, the vignetting rapidly becomes less as the lens is stopped down, and such an arrangement does not necessarily represent an undesirable compromise. The user has a compact camera without excessive vignetting for the aperture ratios that are usually used with modern rapid film and at the same time has high speed, at least for the central part of the picture, for the occasions when it is required. However, when exposures are made on color film with the maximum aperture, such vignetting, because of the generally reduced latitude of color film, may be sufficient to detract from the effectiveness of the picture

\section{System of Stop Calibration that Gives Weight to Vignetting}

The method of stop calibration of section II compensates for the different transmittances of different photographic objectives and insures equivalent exposures at the center of the image field for different lenses when used at the same equivalent aperture ratios. However, it does not distinguish between the behaviors of different lenses that arise because of the differences in vignetting. In figure 5 the arrangement is the

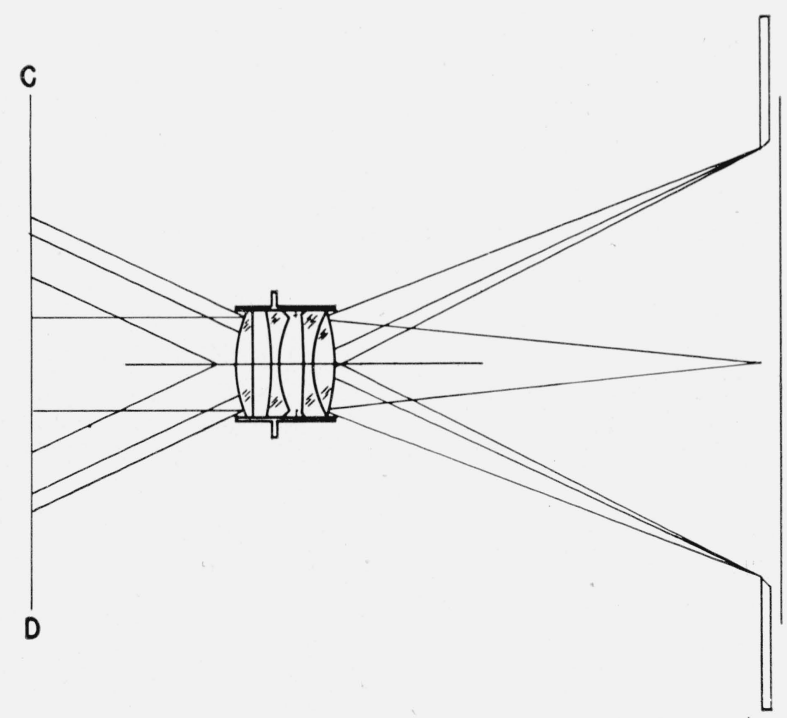

FiguRE 5.-The aperture in plate $G H$ includes the entire field of view utilized when the lens is mounted for use in a camera.

The measurement of luminous flux obtained under this condition is characteristic of the average illumination over the entire field instead of the illumination at the center of the field as obtained by the arrangement of figure 1. same as for figure 1, except that instead of meassuring the illumination of a small area near the axis, the total flux received by an area in the focal plane identical with the picture frame is measured. Similarly, for the comparative measurement with an aperture only, the arrangement of figure 2 is modified to give a measurement of the flux received by the entire field. The equivalent aperture ratios, as before, are considered equal when the two flux measurements are equal. If $e$ of figure 2 and $f$ of figure 6 are not equal, the angular subtenses of the two field-stops at the center of the diaphragm and exit pupil of lenses must be equal when the two measurements of flux are made, and the differences in area should be considered. For photographic purposes, the agreement will be satisfactory, provided that the two field apertures are identical and the value of $e$ and $f$ do not differ by more than 1 percent.

For a motion-picture camera or a miniature camera, the field aperture required is small, and it is generally not too difficult to make the measure as outlined. If the picture frame is large, it may be desirable to reverse the direction of travel of the light by using an integrating sphere to illuminate the picture frame. The total flux is then measured by a photoelectric element with a receiver large enough to receive all the light that passes through the lens. By this reversal of the direction of travel of the light, a photocell with a receiver smaller than otherwise applicable can be used.

If the iris of a lens is calibrated by the two methods, measuring central illumination only and measuring the flux received by the entire field, it will be found that the calibrations, in general, are different. This is understandable because the two methods are based upon different assumptions. In the method first described, a given equivalent aperture ratio corresponds to a definite illumination at the center of the image field. According to the second method, the use of the equivalent aperture ratio corresponds to a given average illumination over the entire field. For a lens that has a large amount of vignetting, a given geometric aperture ratio will correspond to a smaller equivalent aperture ratio than for a lens with less vignetting. Both methods of calibration have advantages, and it is probable that the different standardizing groups interested in photographic procedure should carefully consider the two methods and make recommendations governing their use. 


\section{Calibrations of Stop for Finite Object Distances}

In the foregoing discussion, it has been tacitly assumed that the object to be photographed is at an "infinite" distance and that the image will consequently lie in the focal plane of the lens. This is the basis on which the values of the geometric aperture ratio are engraved on the lens mounts, and it is an entirely satisfactory procedure for a large amount of photographic work. If, for example, the object instead of being at an "infinite" distance is only 10 focal lengths away, the distance from lens to focal plane is only increased by 10 percent of the equivalent focal length and, for many applications, the error in exposure resulting from using the values corresponding to the image in the focal plane will not be excessive.

Lenses for copying purposes and some other types are habitually used with the object distant only a few focal lengths from the lens. In such instances it is highly desirable that the aperture ratios be marked for one or more selected object distances approximating those which will actually be employed in practice. The method of stop calibration can be readily extended to apply to this problem.

Suppose, for example, that the selected object distance is $2 f$, corresponding to the use of the lens for one-to-one copying. Referring to figure 1, plate $G H$ bearing aperture $O$ will be moved back from the lens to the image plane corresponding to one-to-one copying. Referring to figure 2, plane $G H$ will be separated from the diaphragm by approximately the same distance as for the lens. For this particular case, the separation would be twice the equivalent focal length of the lens. With this new spacing, the diameter of aperture $I J$ should be determined, so that $\sin \alpha$ has a value corresponding to a selected aperture ratio, as given in table 1. Measurements are now made as before, the diaphragm setting of the lens being altered until the two flux readings are the same. When equality is obtained, the equivalent aperture ratio of the lens, for the one-to-one ratio, is equal to the geometrical aperture ratio of the diaphragm.

If the iris markings are calibrated in this manner, the lens will give the same exposure for a given equivalent aperture ratio and one-to-one copying as does the same lens or any other lens with the calibration for infinite distances when used on a distant object. For a finite distance, the question again arises as to whether the calibration should be similar to that of section II, with exposure at the center of the field as the criterion, or to the method of section IV, in which the criterion is average exposure over the entire field.

\section{Advantages of Proposed Method of Lens Aperture Calibration and System of Lens Marking}

The foregoing text refers to several published papers dealing with this subject. The method of calibration and marking herein proposed offers advantages not possessed by any of the previously suggested methods:

1. The standard aperture to which reference is made is an aperture of known diameter in a metal plate, and therefore can be readily produced by any laboratory. This facilitates the maintenace of consistent systems of graduation by different laboratories.

2. Each calibration is essentially a substitution method in which the two values of flux to be measured are of approximately the same value. This largely eliminates errors arising from the nonlinearity of response of the photometric apparatus and eliminates the need for carefully calibrated filters.

3. No condenser or collimator system is used. Hence the method does not involve the assumption that the distribution of energy in a collimated beam is uniform.

4. A method of application is proposed that requires no modification of present models of exposure meters. New film-speed tables are required, but presumably the data already in the possession of manufacturers of exposure meters will be sufficient for the preparation of these tables.

5. A system of lens marking is proposed that permits exposure to be determined either by the conventional or new method.

6. An extension of the method of calibration has been given that permits lenses to be calibrated for object distances other than infinite.

7. The calibrated value may be based on brightness of image at the center of the field or on average brightness of image over all the field, whichever may be considered preferable.

Washington, December 23, 1946. 